\title{
Assessment of Bisphenol A (BPA) neurotoxicity in vitro with mouse embryonic stem cells
}

\author{
Nuoya Yin ${ }^{1}$, Xinglei Yao ${ }^{1}$, Zhanfen Qin $^{1}$, Yuan-Liang Wang ${ }^{2}$, Francesco Faiola, ${ }^{1, *}$ \\ 1. State Key Laboratory of Environmental Chemistry and Ecotoxicology, Research Center for Eco-Environmental Sciences, \\ Chinese Academy of Sciences, Beijing 100085, China. E-mail: nyyin@rcees.ac.cn \\ 2. Section of Molecular Biology, University of California at San Diego, La Jolla, CA 92093, USA
}

\section{A R T I C L E I N F O}

Article history:

Received 27 April 2015

Revised 18 June 2015

Accepted 23 June 2015

Available online 7 July 2015

Keywords:

Bisphenol A (BPA)

Stem cell toxicology

Neurotoxicity

Embryonic stem cells (ESCs)

Developmental toxicity

Neural ectoderm

\begin{abstract}
A B S T R A C T
The adverse effects of environmental pollution on our well-being have been intensively studied with many in vitro and in vivo systems. In our group, we focus on stem cell toxicology due to the multitude of embryonic stem cell (ESC) properties which can be exerted in toxicity assays. In fact, ESCs can differentiate in culture to mimic embryonic development in vivo, or specifically to virtually any kind of somatic cells. Here, we used the toxicant Bisphenol A (BPA), a chemical known as a hazard to infants and children, and showed that our stem cell toxicology system was able to efficiently recapitulate most of the toxic effects of BPA previously detected by in vitro system or animal tests. More precisely, we demonstrated that BPA affected the proper specification of germ layers during our in vitro mimicking of the embryonic development, as well as the establishment of neural ectoderm and neural progenitor cells.
\end{abstract}

(C) 2015 The Research Center for Eco-Environmental Sciences, Chinese Academy of Sciences. Published by Elsevier B.V.

\section{Introduction}

During our everyday life we are constantly exposed to many artificial substances created in numerous industrial processes. Many of these materials tend to accumulate in the environment. As a consequence, human exposure to these chemicals, and the potential adverse health effects caused by them, may occur even in the absence of direct use of these industrial products. This has raised a growing concern about the effects of environmental pollution on human health and prompted the urgency and necessity of validated and comprehensive toxicity tests to address the potential hazardousness of these pollutants. Stem cell toxicology is a very powerful alternative to animal tests or traditional in vitro assays because it allows to test in vitro the acute and developmental toxicities of a pollutant of interest, quickly, thoroughly, and cost-effectively (Faiola et al., 2015; Jennings, 2014). In fact, embryonic stem cells (ESCs) offer the advantage that they can be derived easily and also cultured indefinitely in dishes. Therefore, they can be employed without problems for cytotoxicity assays like any other kind of cells. In addition, ESCs can be utilized in developmental toxicity assays. For instance, they can differentiate in vitro as three dimensional aggregates so-called embryoid bodies (EBs) which mimic the early stages of embryonic development in vivo. Moreover, ESCs can virtually differentiate specifically into any type of cells of an adult organism. Those cells can then be used

\footnotetext{
* Corresponding author. E-mail: faiola@rcees.ac.cn (Francesco Faiola).
} 
for cell function toxicity assays as any other derived primary cell type (Wobus and Loser, 2011; Liu et al., 2013; Mori and Hara, 2013).

One of the most studied and dreaded environmental pollutants to date is a chemical called Bisphenol A (BPA). It has been employed since the 1950s to make polycarbonate plastics in a variety of products. BPA has been also used to coat the inner surface of beverage and food containers, regardless of the fact that it has been recognized as an endocrine-disrupting chemical (EDC) since 1936 (Dodds and Lawson, 1936). Indeed, countless studies in the last two decades have documented BPA-related effects on fertility, genital and behavioral abnormalities, heart disease, diabetes, and obesity (Rochester, 2013). However, in those reports, many different in vitro and in vivo systems have been employed, but almost none utilized stem cells. In addition, when BPA was tested with mouse ESCs, little or no toxic effects were detected (Panzica-Kelly et al., 2013; Kong et al., 2013).

In this study, we employed the known developmental toxicant BPA to test its effects on mouse ESCs with our stem cell toxicology system. Contrary to previous reports, we were able to detect BPA toxicity in vitro, particularly towards the neural ectoderm specification.

\section{Materials and methods}

\subsection{Cell culture}

All cell culture reagents and plasticware were purchased from Gibco (Life Technologies, NY, USA) and Corning (USA), respectively, unless otherwise indicated. J1 mouse ES cells were acquired from the Institute of Biochemistry and Cell Biology, Shanghai Institutes for Biological Sciences, Chinese Academy of Sciences. Cells were cultured at $37^{\circ} \mathrm{C}$ in humidified air with $5 \%$ $\mathrm{CO}_{2}$ in high glucose KnockOut DMEM medium supplemented with $15 \%$ fetal bovine serum, $2 \%$ antibiotics $(100 \mathrm{U} / \mathrm{mL}$ penicillin and $100 \mu \mathrm{g} / \mathrm{mL}$ streptomycin), 1\% nucleosides, 1\% glutamine, $1 \%$ non-essential amino acids, $10^{-4} \mathrm{~mol} / \mathrm{L} \beta$-mercaptoethanol and $10^{3} \mathrm{U} / \mathrm{mL}$ leukemia inhibitory factor (LIF) (Merck Millipore, Darmstadt, Germany). All plates were coated with $0.1 \%$ gelatin (Sigma-Aldrich, MO, USA) before use.

\subsection{Cytotoxicity assay}

For cytotoxicity assays, mESCs were pre-incubated with 1-10 $\mu \mathrm{mol} / \mathrm{L}$ BPA (or DMSO solvent control) for $24 \mathrm{hr}$, then seeded in 96-well gelatin-coated plates, and incubated with BPA/DMSO for seven days. Cell viability was determined by the AlamarBlue (AB, Sigma-Aldrich, MO, USA) fluorescence assay. Briefly, cells were incubate with the AlamarBlue reagent for two hours at $37^{\circ} \mathrm{C}$, and fluorescence was measured in a multiwall fluorometric reader (ThermoFisher Scientific, MA, USA) with an excitation wavelength of $530 \mathrm{~nm}$ and an emission wavelength of $590 \mathrm{~nm}$.

\subsection{Alkaline phosphatase (AP) staining}

To visually detect undifferentiated mESCs, an AP staining kit (Sigma-Aldrich, MO, USA) was used according to the manufacturer's instructions.

\subsection{Embryoid body (EB) differentiation assay}

For EB differentiation assays, $4 \times 10^{6} \mathrm{mESCs}$ were resuspended by trypsinization and seeded in $10-\mathrm{cm}$ petri dishes in ES medium without LIF, to allow aggregation and formation of EBs. Media were replaced every other day and samples collected at days $0,2,4,6,9,12$, and 20, for RNA extraction and qRT-PCR measures. One day before the start and along the differentiation process, cells were incubated with $10 \mu \mathrm{mol} / \mathrm{L}$ BPA or DMSO control.

\section{5. mESC differentiation into neural progenitor cells (NPCs)}

mESCs were pre-treated with $10 \mu \mathrm{mol} / \mathrm{L}$ BPA or vehicle control for $24 \mathrm{hr}$, and then allowed to form EBs as described above. At day 4 of EB formation, a final concentration of $5 \mu \mathrm{mol} / \mathrm{L}$ retinoic acid (RA, Sigma-Aldrich, MO, USA) was added to the medium to stimulate neural ectoderm specification. At day 8 , EBs were dissociated into single cells by trypsinization and passage through a $40 \mu \mathrm{m}$ nylon cell strainer (BD, USA), and seeded into plates coated with laminin/poly-L-lysine (Roche, USA, and Sigma-Aldrich, USA, respectively) in N2 medium (DMEM-F12, N2 supplement, 1\% GlutaMAX, and 2\% Pen/Strep antibiotics). Media were replaced after $2 \mathrm{hr}$. Samples were collected at days $0,4,8$, and 10 for RNA extraction and qRT-PCR analyses.

\subsection{Adherent cell neuroectoderm differentiation}

For the differentiation of mESCs into neural ectoderm in monolayer conditions, cells were pretreated with $10 \mu \mathrm{mol} / \mathrm{L}$ BPA or DMSO control as above. Then, RA was added to the medium to stimulate neuroectoderm differentiation. Samples were collected every other day from day 0 to day 10 for RNA extraction and gene expression analyses by qRT-PCR.

\subsection{RNA extraction and $q R T-P C R$ analyses}

Total RNA was extracted with Trizol ( Life Technologies, NY, USA), following the instructions of the manufacturer. RNA integrity was assessed by agarose gel electrophoresis and ethidium bromide staining. RNAs were converted to cDNAs with the PrimeScript RT Master Mix Kit (Takara, Japan) according to the manufacturer's recommended procedures. qPCR was performed with the SYBR Premix Ex Taq kit (Takara, Japan). Cycling conditions were executed forty times, except initial denaturation and final cycle, as following: initial denaturation, $95^{\circ} \mathrm{C} 30 \mathrm{sec}$; denaturation, $95^{\circ} \mathrm{C} 5 \mathrm{sec}$; annealing, $60^{\circ} \mathrm{C} 30 \mathrm{sec}$; final cycle, $95^{\circ} \mathrm{C} 5 \mathrm{sec}, 60^{\circ} \mathrm{C} 1 \mathrm{~min}, 95^{\circ} \mathrm{C} 30 \mathrm{sec}$. The comparative $\mathrm{C}_{t}$ methods was used to calculate the relative gene expression normalized to the gapdh gene. Measures were obtained in triplicates. Results are shown as mean \pm standard deviation (SD). Primers employed are listed in Table 1.

\subsection{Statistical analysis}

Statistical significance was determined by multiple t-test using the Holm-Sidak method, with alpha $=5.000 \%$. Each row was analyzed individually, without assuming a consistent SD. 


\section{Table 1 - List of primers used in qPCR assays.}

\begin{tabular}{|c|c|c|c|}
\hline Primer & Sequence $\left(5^{\prime}-3^{\prime}\right)$ & Primer & Sequence $\left(5^{\prime}-3^{\prime}\right)$ \\
\hline gapdh (F) & CGTCCCGTAGACAAAATGGT & brachyury (F) & GTCTAGCCTCGGAGTGCCT \\
\hline gapdh (R) & TTGATGGCAACAATCTCCAC & brachyury (R) & CCATTGCTCACAGACCAGAG \\
\hline $\operatorname{pax6}(\mathrm{F})$ & CGGGACTTCAGTACCAGGG & nestin $(F)$ & AGATCGCTCAGATCCTGGAA \\
\hline $\operatorname{pax6}(\mathrm{R})$ & CTTCATCCGAGTCTTCTCCG & nestin (R) & GAGTTCTCAGCCTCCAGCAG \\
\hline fgf5 (F) & GCTGTGTCTCAGGGGATTGT & $\operatorname{sox1}(\mathrm{F})$ & AGTGGAAGGTCATGTCCGAG \\
\hline fgf5 (R) & ACAGTCATCCGTAAATTTGGC & $\operatorname{sox} 1(\mathrm{R})$ & TGTAATCCGGGTGTTCCTTC \\
\hline gata6 (F) & TACACAAGCGACCACCTCAG & $\operatorname{sox} 3(\mathrm{~F})$ & ACTGGAAACTGCTGACCGAT \\
\hline gata6 (R) & TGTAGAGGCCGTCTTGACCT & $\operatorname{sox} 3(\mathrm{R})$ & CCGGTACTTGTAGTCCGGGT \\
\hline sox17 (F) & TGGAACCTCCAGTAAGCCAG & krt14 (F) & GGCCCACTGAGATCAAAGAC \\
\hline sox17 (R) & TCAGATGTCTGGAGGTGCTG & krt14 (R) & GATCTGCAGGAGGACATTGG \\
\hline hand1 (F) & TGAACTCAAAAAGACGGATGG & $c d \times 2(F)$ & GAAACCTGTGCGAGTGGATG \\
\hline hand1 (R) & СТTTAАTССТСТTCTCGCCG & $c d x 2(\mathrm{R})$ & TCTGTGTACACCACCCGGTA \\
\hline
\end{tabular}

F: forward primer; R: reverse primer.

\section{Results and discussion}

\subsection{BPA cytotoxicity}

In order to employ appropriate BPA concentrations during our developmental toxicity assays, we first determined the cytotoxicity properties of BPA with mESCs. Since our differentiation procedures would last at least one week, we measured BPA-dependent acute toxicity upon seven-day incubation with the chemical. Fig. 1a (left panel) shows that mESC viability was not significantly affected by BPA concentrations ranging from $1 \mathrm{nmol} / \mathrm{L}$ to $1 \mu \mathrm{mol} / \mathrm{L}$, as compared to DMSO solvent control. To assess whether ESCs were not only alive but also in the undifferentiated state, we stained the cells with alkaline phosphatase (AP) at the end of the cytotoxicity assay. Only live and self-renewing cell would stain dark red, while differentiating cells would stay unstained or only partially stained. As depicted in Fig. 1b (top panel), similar amounts of ESC colonies were stained red for AP in all wells, suggesting similar levels of undifferentiated ESCs upon incubation with BPA or DMSO. We also incubated our cells with $10 \mu \mathrm{mol} / \mathrm{L}$ BPA, or solvent vehicle, for a week and measured cell viability and AP staining. Fig. 1a (right panel) and B (bottom panel) shows no effects on cell viability and self-renewal at this BPA concentration. These results agree with the two previously published studies (Panzica-Kelly et al., 2013; Kong et al., 2013). We also passaged our ESCs in the presence of $10 \mu \mathrm{mol} / \mathrm{L}$ BPA for several times, and observed no noteworthy effects on cell viability and self-renewal (data not shown). Thus, we were confident we were using concentrations of BPA far from lethal.

\subsection{BPA affected mESC embryoid body (EB) differentiation}

To test if BPA behaved as a toxicant in our stem cell system, we mimicked embryonic development in vitro by differentiating mESCs via EB formation. In fact, when ESCs aggregate in suspension to form EBs in differentiating conditions, they start specifying the three primary germ layers, endoderm, mesoderm, and ectoderm, similarly to what would happen in vivo. ESCs were pre-incubated with $10 \mu \mathrm{mol} / \mathrm{L}$ BPA, or vehicle, for one day, and then allowed to aggregate in suspension in ES medium deprived of LIF (day 0). EB samples were then collected approximately every other day for RNA extraction and qRT-PCR analyses. We then checked for the expression of markers for endoderm (gata6, sox17), mesoderm (brachyury (T), hand1), ectoderm (fgf5, krt14), and trophectoderm (cdx2). As shown in Fig. 2a, there was a slight reduction in the expression of the two endoderm markers tested when cells where incubated with BPA, as compared to DMSO control. The trophectoderm marker cdx2 seemed to be affected by BPA exposure as well, with an initial BPA-dependent increase (day 4 and day 9) followed by a pronounced reduction at days 12 and 20 of EB formation (Fig. 2d). This interesting behavior is under further investigation and will be described in a future study. Conversely, mesoderm specification appeared not to be altered by the presence of BPA (Fig. 2c). This could explain why in a previous study (Kong et al., 2013) they did not detect any noteworthy BPA toxicity, since they only looked at MESC differentiation into beating cardiomyocytes, which had derived from mesoderm. When we analyzed BPA effects on ectoderm specification, we did not notice any noteworthy down-regulation in the expression of the early ectoderm marker fgf5 (Fig. 2b). Interestingly, fgf5 exhibited a quick up-regulation at early stages (up to day 4), and gradually decreased afterward, and BPA treatment slightly increased its transcription. On the contrary, the expression of the non-neural and late ectoderm marker krt14 (one of the keratins in skin epithelia) appeared to be negatively affected by the presence of BPA, at least at day 20 of the differentiation procedure. These observations suggest that BPA may stimulate the initial specification of ectoderm, but negatively affects the later generation of tissues, such as the skin, which are derived from ectoderm.

Numerous studies have demonstrated the BPA neurotoxic effects with in vitro and in vivo systems (Beronius et al., 2013). Thus, we examined the neural ectoderm specification during our EB differentiation assays upon incubation with BPA. Our molecular read-out was the expression of four neuroectoderm markers: sox1, pax6, sox3, and nestin. As depicted in Fig. 3, all four genes showed reduced expression in the BPA-treated samples, relative to DMSO control ones. This implied a BPA-dependent neurotoxicity during embryonic development, and that our stem cell toxicology system was able to detect it. 

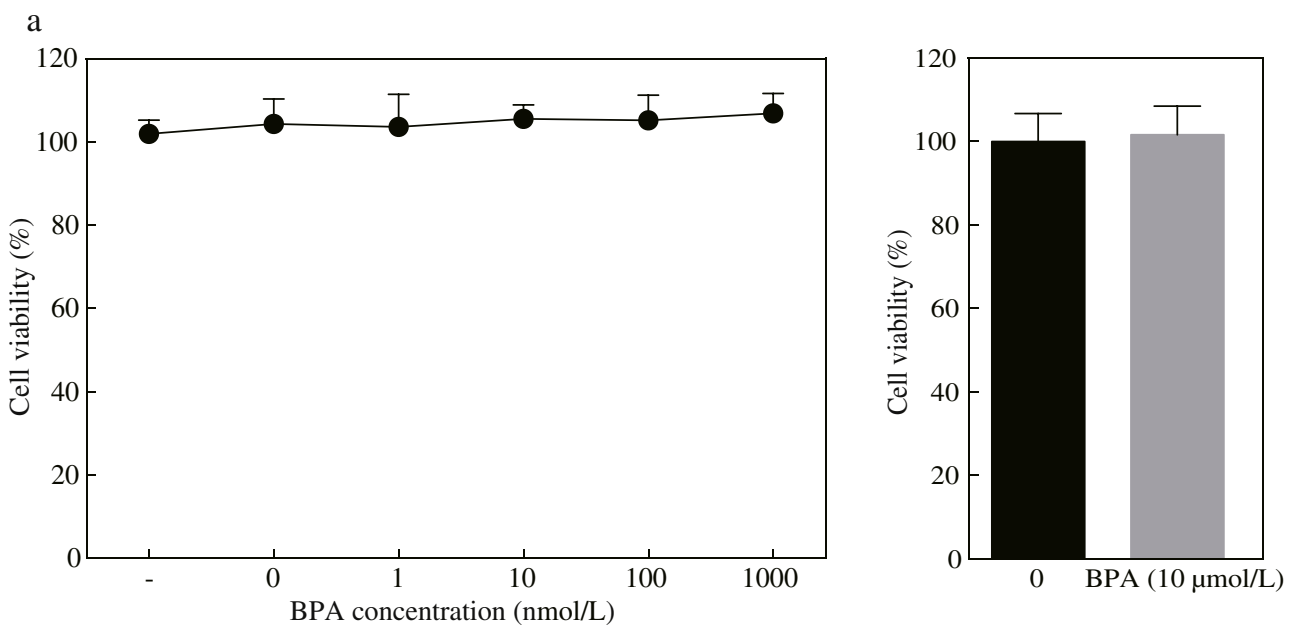

$\mathrm{b}$

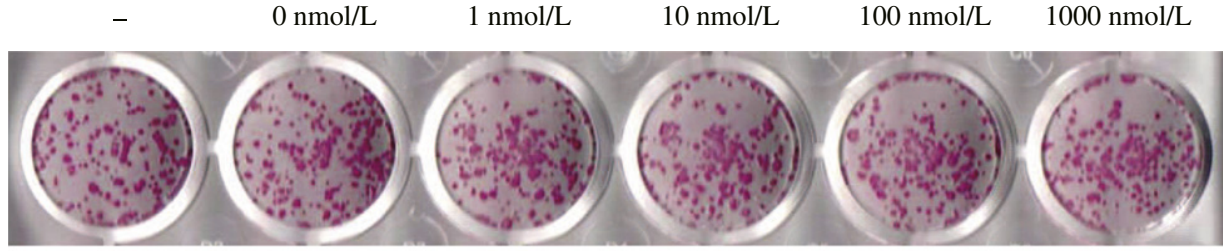

$0 \mu \mathrm{mol} / \mathrm{L}$

$10 \mu \mathrm{mol} / \mathrm{L}$

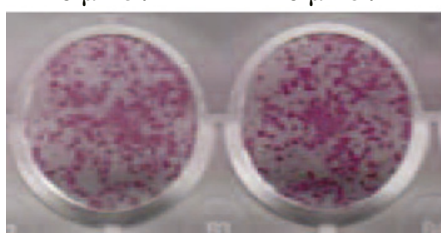

Fig. 1 - BPA did not alter mESC viability at the concentrations tested. (a) Viability plot for cells incubated with 0-1000 nmol/L BPA (left panel), or $10 \mu \mathrm{mol} / \mathrm{L}$ BPA (right panel) for seven days. (b) Alkaline Phosphatase staining at day 7 of representative wells for cells incubated with the indicated concentrations of BPA. - indicates no treatment; $0 \mathrm{nmol} / \mathrm{L}$ refers to DMSO only.

\subsection{BPA significantly impaired EB-based neural differentiation of mESCs}

Intrigued by the substantial alteration of all the neural ectoderm markers checked during mESC EB differentiation in the presence of BPA, we decided to utilize a differentiation procedure to specifically differentiate ESCs into neuroectoderm first, and then into neural progenitor cells (NPCs). This would allow a more precise assessment of BPA neurotoxic abilities. Thus, we incubated the cells with $10 \mu \mathrm{mol} / \mathrm{L} \mathrm{BPA}$, or DMSO solvent, and add retinoic acid (RA) starting from day 4 during the EB differentiation process to induce neural ectoderm. At day 8, we dissociated the EBs and transferred the cells to plates coated with laminin/poly-L-lysine to culture NPCs. We then collected samples at different time points and checked for the expression of specific neural markers. As depicted in Fig. 4a, all the markers checked were significantly affected by BPA incubation, as compared to DMSO control. It is also noteworthy that a higher expression of the markers was observed in this differentiation process as compared with the global EB differentiation of Fig. 3. These results suggested an effect of RA in neuroectoderm formation. We also looked at the early ectoderm marker fgf5 and the late non-neural ectoderm gene krt14. Fgf5 was, as expected, activated only at early stages (up to day 4), and became quickly repressed afterward. Exposure to BPA reduced its expression. Conversely, krt14 was never expressed (Fig. 4b). These data clearly confirm the deleterious effects of BPA on the neural lineage specification we observed during the global differentiation of mESCs.

\subsection{Adherent neuroectoderm differentiation of mESCs was also compromised by BPA}

To differentiate mESCs into neural ectoderm, we employed an alternative and simplified protocol based on monolayer conditions. Also in this case, we removed LIF and added RA to stimulate the differentiation. Although the efficiency of this procedure is generally not as good as the one from the EB-based process, the majority of the cells differentiate towards neuroectoderm. Upon $10 \mu \mathrm{mol} / \mathrm{L}$ BPA incubation, we determined the levels of expression of three markers, sox1, pax6, and nestin. Fig. 5 shows that sox 1 and pax6 expression were impaired in the presence of BPA, as compared to DMSO control, while transcription of nestin was not affected by the chemical. These results confirmed the effects of BPA on the 
a) Endoderm

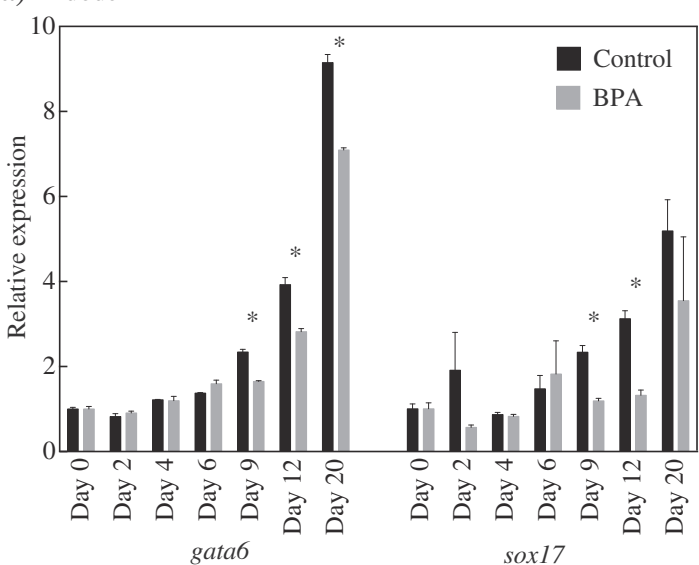

c) Mesoderm

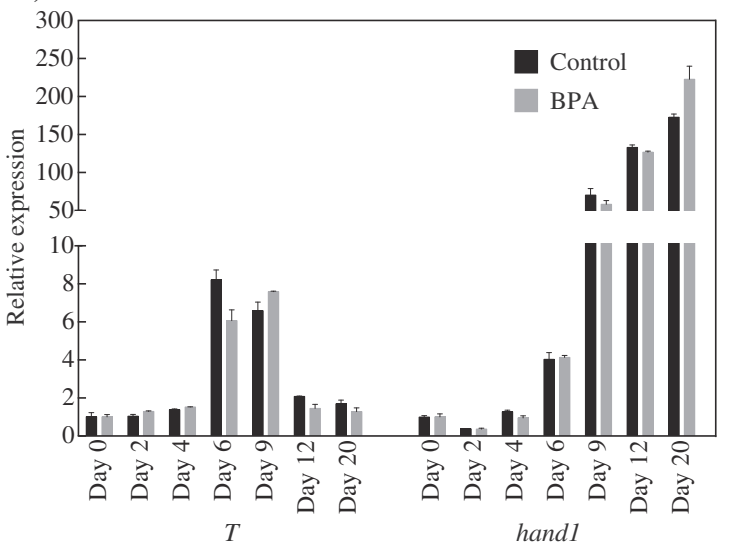

b) Ectoderm

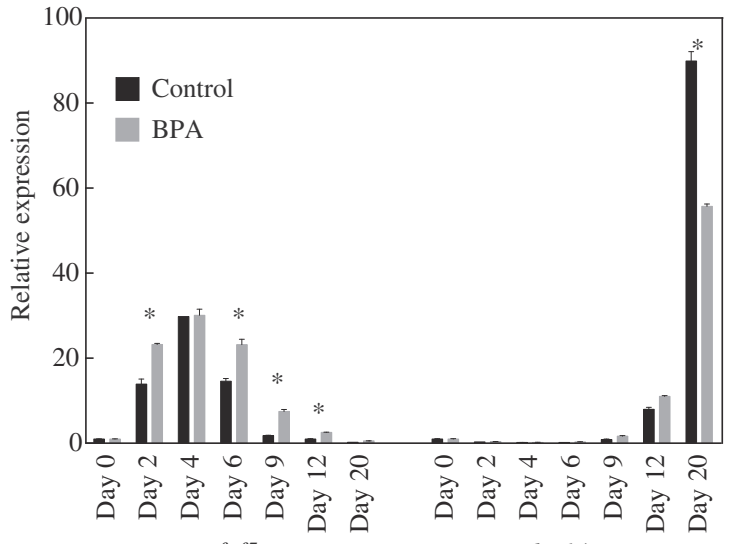

krt14

d) Trophectoderm

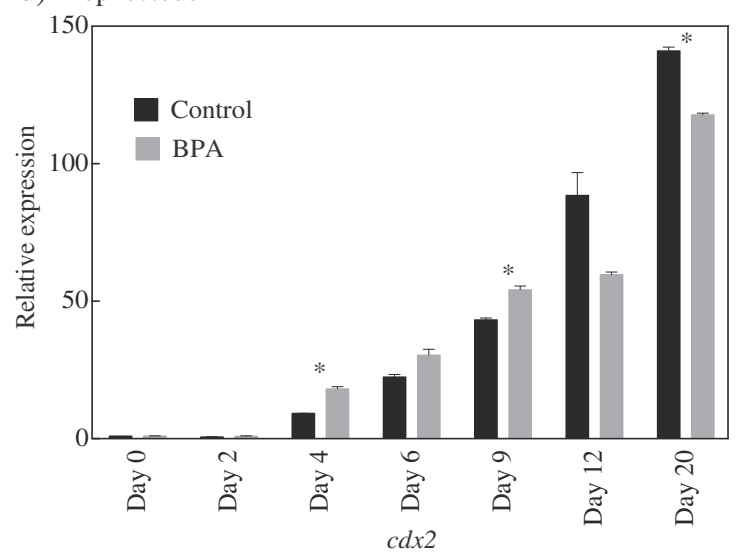

Fig. 2 - Expression profiling of lineage specific markers during embryoid body-based differentiation of mESCs upon incubation with BPA or DMSO vehicle. Specific marker expression for (a) endoderm (gata6, sox17), (b) ectoderm (fgf5, krt14), (c) mesoderm (T, hand1), and (d) trophectoderm (cdx2), respectively. ${ }^{*}$ indicates a statistical significant difference in gene expression between BPA and control samples.

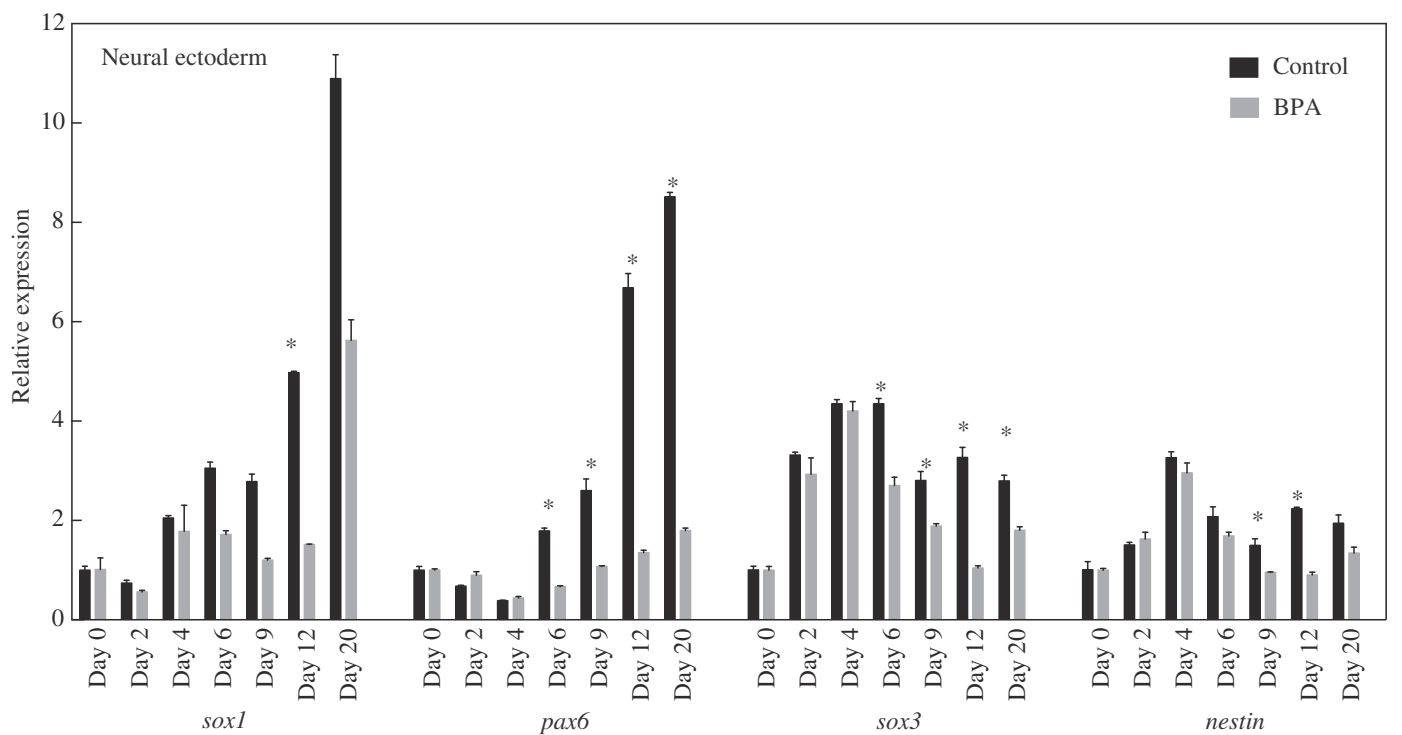

Fig. 3 - BPA treatment affected neural ectoderm specification during EB differentiation. Expression of four typical neuroectoderm markers was analyzed by QRT-PCR. * indicates a statistical significant difference in gene expression between BPA and control samples. The statistical analysis for sox1 day 6, 9, and 20 samples was not performed because the number of replicates was equal to 2 . 
a) Neural ectoderm

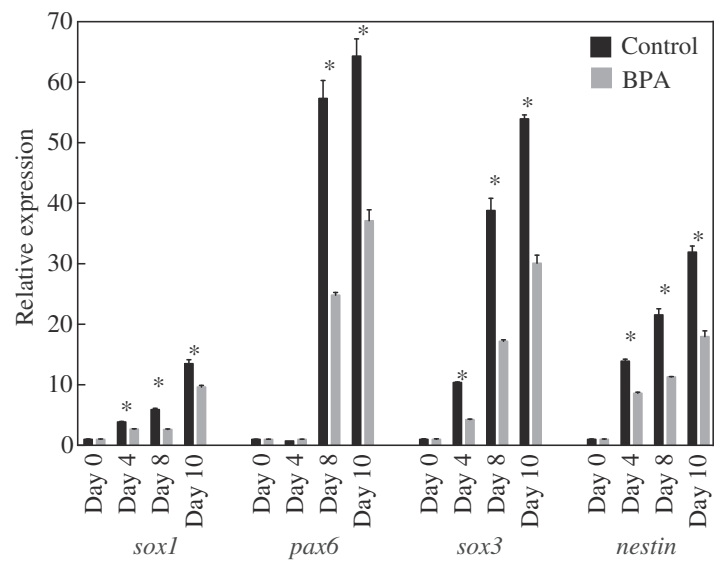

b) Non-neural ectoderm

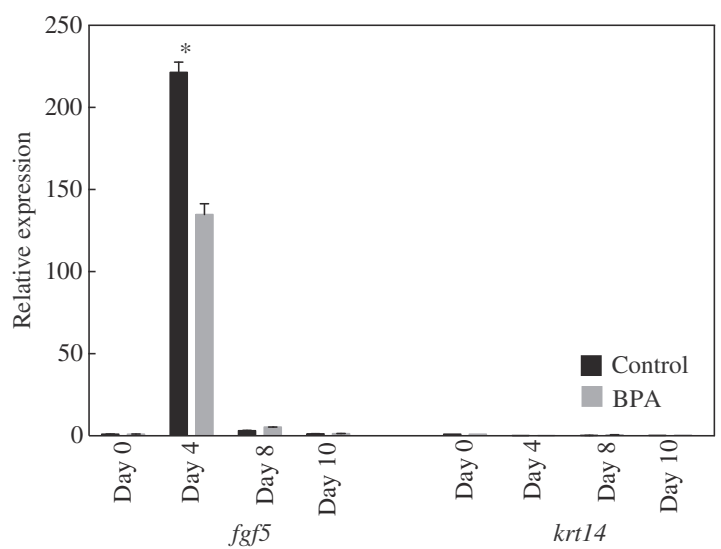

Fig. 4 - Differentiation of mESCs towards neural progenitors cells (NPCs) was compromised by BPA. (a) Expression of four typical neural markers: sox1, pax6, sox3, and nestin upon BPA or DMSO treatment; (b) expression of the early ectoderm marker fgf5, and the non-neural ectoderm marker krt14, upon treatment with BPA or DMSO. * indicates a statistical significant difference in gene expression between BPA and control samples.

expression of the two transcription factors sox1 and pax6, similar to what we observed above. The discrepancy about BPA effects on nestin expression between the two differentiation procedures can be explained by the fact that the adherent differentiation, although quicker and less technically challenging, is not as efficient and specific as the one based on EBs. As a result, more elusive gene expression differences may not be easily detected.

\section{Conclusions}

In this study, we demonstrated that stem cell toxicology could be a powerful system to detect the developmental toxicity of environmental pollutants. We employed BPA as a test chemical owing to its toxicity established with many other in vitro and in vivo systems. With our EB-based differentiation process that mimics in vitro embryonic development, we showed that BPA slightly altered the expression of endoderm and trophectoderm markers, and significantly impaired the neural ectoderm specification. The neurotoxic effects of BPA were also evident in our two additional differentiation procedures specifically directed towards neural ectoderm and/or neural progenitor cells, via EB formation or in monolayer conditions. In sum, we proved that our stem cell toxicology system could serve as a noteworthy improvement over traditional stem cell toxicity assays, which were previously shown to detect either no BPA toxicity (Panzica-Kelly et al., 2013), or just slight embryotoxic effects (Kong et al., 2013). Compared to the first study, our combination of several differentiation procedures (EB-based or in monolayer

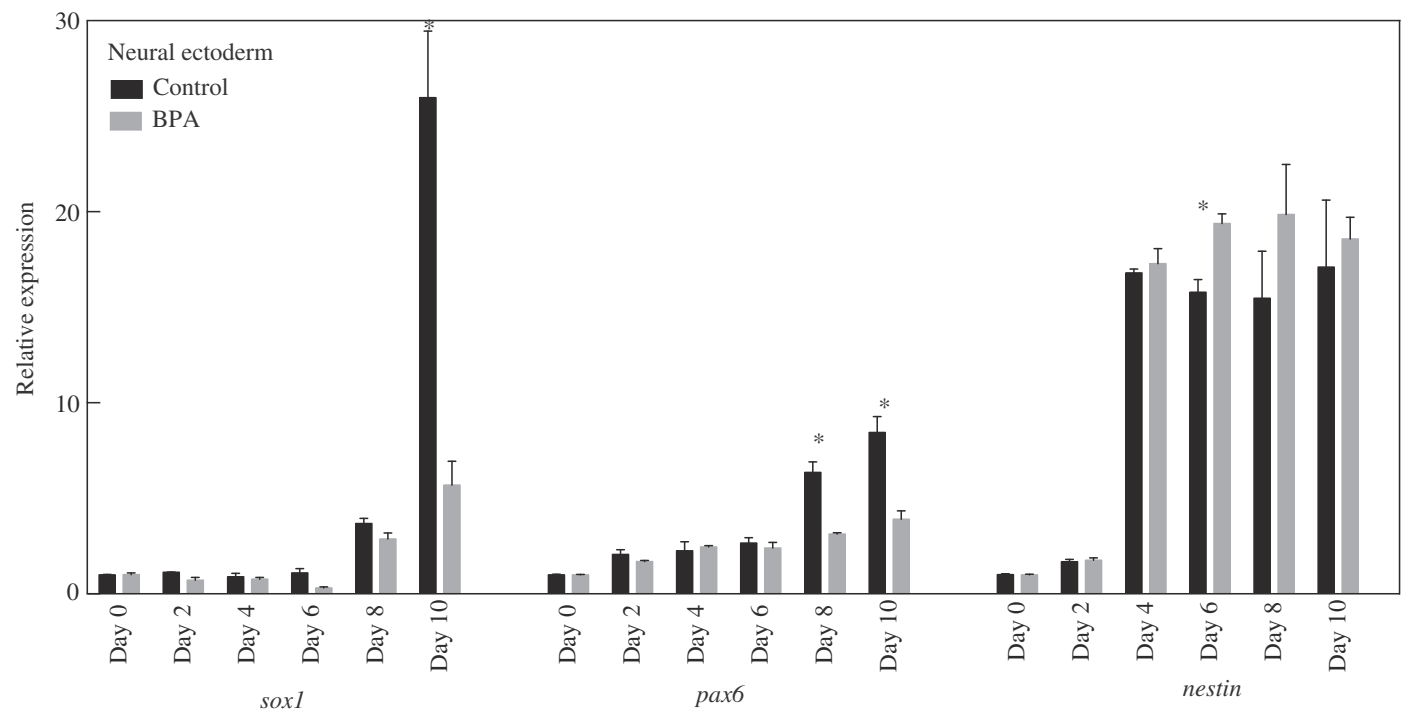

Fig. 5 - BPA impaired the neural ectoderm differentiation of mESCs in monolayer conditions. The time course expression profiling of the three markers sox1, pax6, and nestin is shown. ${ }^{*}$ indicates a statistical significant difference in gene expression between BPA and control samples. 
conditions) with gene expression analyses over a time course, proved to be more sensitive and reflective of the physiological toxicity effects of BPA, not detected by single-point analyses (Panzica-Kelly et al., 2013). In addition, our approaches were able to examine the effects of BPA on the development of all three germ layers, instead of just one, mesoderm (Kong et al., 2013). Most importantly, our methodology was much more sensitive and efficient in detecting BPA as a toxicant by using concentrations well below lethality. It also argues against the choice of a single time point during the differentiation process to assess toxicity. The improved sensitivity and efficiency established by our system will be of tremendous use for testing the effects of other hazardous chemicals on human health.

\section{Acknowledgments}

This work was supported by a Chinese Academy of Sciences (CAS) Strategic Leading Science \& Technology Program grant (XDB14040301), and by the Hundred Talent Program of CAS (121311ZXPP2014004) at the Research Center for Eco-Environmental Sciences (RCEES), CAS. We thank Prof. Guibin Jiang for suggestions.

\section{R E F E R E N C E S}

Beronius, A., Johansson, N., Ruden, C., Hanberg, A., 2013. The influence of study design and sex-differences on results from developmental neurotoxicity studies of bisphenol A: implications for toxicity testing. Toxicology 311 (1-2), 13-26.
Dodds, E.C., Lawson, W., 1936. Synthetic oestrogenic agents without the phenanthrene nucleus. Nature 137, 996.

Faiola, F., Yin, N., Yao, X., Jiang, G., 2015. The rise of stem cell toxicology. Environ. Sci. Technol. 49 (10), 5847-5848.

Jennings, P., 2014. The future of in vitro toxicology. Toxicol. in Vitro http://dx.doi.org/10.1016/j.tiv.2014.08.011 (available on line).

Kong, D., Xing, L., Liu, R., Jiang, J., Wang, W., Shang, L., et al., 2013. Individual and combined developmental toxicity assessment of bisphenol A and genistein using the embryonic stem cell test in vitro. Food Chem. Toxicol. 60, 497-505.

Liu, W., Deng, Y., Liu, Y., Gong, W., Deng, W., 2013. Stem cell models for drug discovery and toxicology studies. J. Biochem. Mol. Toxicol. 27 (1), 17-27.

Mori, H., Hara, M., 2013. Cultured stem cells as tools for toxicological assays. Journal of bioscience and bioengineering. J. Biosci. Bioeng. 116 (6), 647-652.

Panzica-Kelly, J.M., Brannen, K.C., Ma, Y., Zhang, C.X., Flint, O.P., Lehman-McKeeman, L.D., et al., 2013. Establishment of a molecular embryonic stem cell developmental toxicity assay. Toxicol. Sci. 131 (2), 447-457.

Rochester, J.R., 2013. Bisphenol A and human health: a review of the literature. Reprod. Toxicol. 42, 132-155.

Wobus, A.M., Loser, P., 2011. Present state and future perspectives of using pluripotent stem cells in toxicology research. Arch. Toxicol. 85 (2), 79-117. 